\title{
Venetoclax and Azacitidine Therapy in Elder Acute Myeloid Leukemia: A Retrospective Evaluation of Real-World Experience
}

\author{
Rong-Hua Hu \\ Xuan Wu Hospital of the Capital Medical University \\ Li Su \\ Xuan Wu Hospital of the Capital Medical University \\ Xiao-Xi Lan \\ Xuan Wu Hospital of the Capital Medical University \\ Xiao-Li Chang \\ Xuan Wu Hospital of the Capital Medical University \\ Wu-Han Hui \\ Xuan Wu Hospital of the Capital Medical University \\ Hong Zhao \\ Xuan Wu Hospital of the Capital Medical University \\ Yi-Xian Guo \\ Xuan Wu Hospital of the Capital Medical University \\ Guo-Xiang Wang \\ Xuan Wu Hospital of the Capital Medical University \\ Wan-Ling Sun ( $\square$ authorwanlingsun@xwhosp.org ) \\ Xuan Wu Hospital of the Capital Medical University
}

Research Article

Keywords: venetoclax, azacytidine, elder, relapsed and refractory, leukemia

Posted Date: August 20th, 2021

DOI: https://doi.org/10.21203/rs.3.rs-738145/v1

License: @ (1) This work is licensed under a Creative Commons Attribution 4.0 International License. Read Full License 


\section{Abstract}

Background $\mathbb{T}$ To investigate the efficacy of venetoclax combined with azacytidine in the treatment of elderly patients with relapsed and refractory (R/R) acute myeloid leukemia囚AML).

Methods $₫$ The clinical data of 9 elderly AML patients over 65 years old, including 5 with R/R AML, using venetoclax and azacytidine were retrospectively analyzed.

Results $₫$ Six males and 3 females with a median age of 71 years were included in this study, of which four patients had at least one relapse, and one patient did not get go into remission after 4 cycles of azacytidine monotherapy, deeming it refractory. Four patients had AML with myelodysplasia-related changes (AML-MRC). After 1 to 13 cycles of treatment using venetoclax and azacytidine, one of the 9 patients died early due to long duration of neutropenia and severe pulmonary infection caused by drugs. and six of the remaining 8 patients obtained complete response or complete response with incomplete hematologic recovery (CR/ CRi), including five R/R patients. One patient did not respond to treatment after two cycles. For the side effects of the treatment, granulocytopenia occurred in all patients, and neutropenia occurred in 8 patients, lasting for an average of 10.5 (6-15) days and was most obvious in the second to third week of treatment. Three patients with TP53 gene mutation positive had following different outcomes. One relapsed patient achieved progression free remission (PFS) for 16 months up to date, and a second patient achieved complete remission but relapsed two months thereafter. Another patient had complete remission in myelology for 4 months, but the variant allele fraction value (VAF) gradually increased, indicative that the disease was about to progress.

ConclusionखVenetoclax combined with azacytidine regimen in elderly patients is an effective and well tolerated rescue scheme for R/R AML.The patients with TP53 mutation with lower VAF may be benifit from Venetoclax and azacytidine. Severe infection caused by neutropenia is an adverse reaction worthy of attention in the treatment process of the regimen.

\section{Background}

Acute myeloid leukemia (AML) is one of the malignant tumors of hematopoietic system. It is the most common type of leukemia in adults.

With the continuing aging of the world population, the median age of AML onset has risen to 68 years old ${ }^{(1)}$. Most elderly AML patients cannot tolerate intensive therapy or the conventional combination of cytarabine with anthracycline $(7+3$ regimen) because of many serious treatment-related complications and poorly reserved organ function. As a result, the complete remission rate (CR) in this population is low, the duration of remission (DOR) is short, the early mortality is high, and the overall prognosis is poor. Appelbaum et al. (2006) found that the elderly patients aged 66-75 years with performance status (PS) $\geq 2$ points had a $31 \%$ likelihood of dying within 30 days of initiating induction chemotherapy, and a remission rate far lower than that in adult patients (2). The median overall survival (OS) of AML patients over 65 years old in the United States from 2000 to 2016 was estimated to be 2.67 months, and the one-year survival rate of the same group was only $21.8 \%{ }^{(3)}$.

With the development of tumor epigenetics, it has been found that abnormal DNA methylation plays an important role in the occurrence and development of elder AML. In recent years, studies have shown that hypomethylation agents (HMA) have advantages over conventional chemotherapy for elderly leukemia patients. A phase III clinical trial (DACO-016) compared the efficacy of the HMA, decitabine with traditional regimen in the treatment of elderly patients with AML. A total of 485 patients (median age 73 years) were enrolled. The complete response or complete response with incomplete hematologic recovery CR / CRi rates of the two groups were $17.8 \%$ and $7.8 \%$, and the median overall survival time was 7.7 months and 5.0 months, respectively $₫ p \otimes 0.108 \llbracket(4)$. In the past several years, low-intensity chemotherapy including HMA has become the standard treatment for elderly AML patients who are not suitable for high-intensity

chemotherapy ${ }^{(5)}$. However, because HMA is unable to clear leukemic stem cells, AML often relapses after drug withdrawal and needs long-term maintenance. Because of these shortcomings, new targeted drugs have been emerging.

Venetoclax (VEN)『a selective B cell leukemia/lymphoma-2 (Bcl-2) inhibitor, can directly bind to Bcl-2 protein, trigger mitochondrial outer membrane permeability and caspase activation, help restore the process of apoptosis, selectively kill AML cells, and increase the sensitivity of AML cells to chemotherapy drugs ${ }^{(6,7)}$.As a single agent for patients with R/R AML, VEN has demonstrated clinical efficacy, but responses were modest and short-lived. The leukemia-free survival and OS were 2.3 months and 4.7 months, respectively ${ }^{(8)}$. It combined with azacytidine, has been shown to have efficacy in newly diagnosed elderly AML patients ${ }^{(9)}$. Foreign clinical trials have tried to use VEN and HMA in elderly patients with relapsed and refractory (R/R) leukemia(10). In China, most elderly unfit patients have to receive single symptomatic and supportive treatment. Thus far, no large-scale case study has been reported in China using VEN combined with azacytidine(AZA) in elderly patients with R/R AML. To investigate the safety and efficacy of this combinatorial therapy in R/R elderly AML patients, we retrospectively analyzed the clinical data on the efficacy and side effects of this regimen in elder AML patients treated with AZA+VEN in the past two years at the Department of Hematology, Xuanwu Hospital, Capital Medical University.

\section{Cases And Methods}

1. Cases: Nine elderly AML patients from December 2018 to July 2021 in our department were treated with AZA+VEN. All patients were diagnosed through examining bone marrow cell morphology, histochemical staining based on the FAB classification. Clinical and laboratory data were collected at initiation of AZA+VEN therapy

2. Diagnostic criteria, Response criteria and survival: Response to venetoclax therapy was determined by using the 2017 European LeukemiaNet (ELN) response criteria (11). The overall response rate (ORR) was defined as the combination of CR/ CRi. OS was calculated from cycle 1 day 1 of therapy until death or time of last follow-up. 
3. Flow cytometric immunophenotype analysis: heparin-anticoagulated bone marrow $(2 \mathrm{~mL})$ was aspirated from 9 patients and the immunophenotype was examined and analyzed by flow cytometry prior to VEN+AZA treatment.

4. Cytogenetic analysis: Heparin-anticoagulated bone marrow $(4 \mathrm{~mL})$ were collected, and chromosomes were prepared by 24-hour culture method. Gbanding technique was employed to stain the chromosomes and the karyotype was analyzed under the microscope and the nomenclatures were written according to International System for Human Cytogenetic Nomenclature ((ISCN2016).

5. Gene mutation detection: DNA was extracted from bone marrow cells anticoagulated with EDTA. Forty-two genes were detected by AML / MDS secondgeneration sequencing chip of Shanghai Yuanqi life science and Technology Co., Ltd.,( see Table1).The library was constructed by PCR amplification following the instruction from the manufacturer. The hot spots of 42 exons were sequenced by Illumina sequencer. The sequencing results were analyzed by sequencer 4.7 software.

Table1

\begin{tabular}{|c|c|}
\hline DNA methylation regulation related genes & 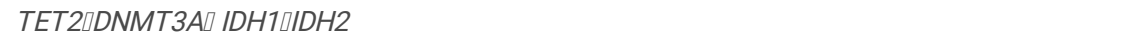 \\
\hline Histone regulation related genes & $E Z H 2 \square A S X L 1 \square P P M 1 D \square R A D 21 \square S M C 1 A \square S M C 3$ \\
\hline splicing factor related genes & 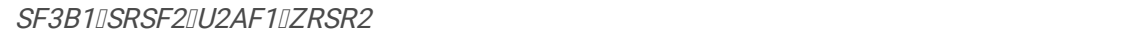 \\
\hline signal transcription related genes & 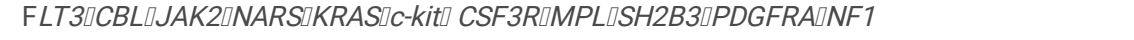 \\
\hline transcription factor related genes & 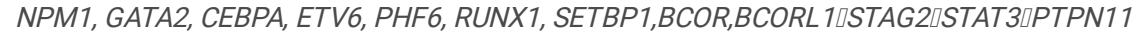 \\
\hline DNA repair related genes & TP53, WT1 \\
\hline others & CALR】PIGA】KMT2A \\
\hline
\end{tabular}

6. Treatment regimen: azacytidine: $75 \mathrm{mg} / \mathrm{m}^{2}$ / d, subcutaneous injection in 2-3 sites for 7 days; VEN: $100 \mathrm{mg}$ on the first day; $200 \mathrm{mg}$ on the second day; 400 $\mathrm{mg}$ from the third day to the 28 th day to complete the 28-day treatment course. The regimen was administered 30 minutes after meals as scheduled. If a CYP3A4 inhibitor, such as fluconazole and posaconazole, was used to treat the accompanied fungal infection, the dosage of VEN was reduced to half, or $1 / 4$, respectively. ${ }^{(12)}$

7. Follow up: The endpoint of follow-up was Jul 1, 2021. Follow-up was conducted by phone calls and/or medical record inquiry.

\section{Results}

1.Clinical data: There were 6 males and 3 females with a median age of 71 (65-82) years old. The PS scores were $\geq 2$. Among them, two patients had secondary recurrence, two patients had their first recurrence, and five patients had AML with myelodysplastic related changes (AML-MRC). Eight patients had at least one cycle of AZA or combined with low-dose chemotherapy before the application of VEN; 3 patients had TP53 gene mutation, of which 2 patients had complex chromosome karyotype (see table 2); 1 patient had FLT3-ITD gene mutation, 3 patients had DNMT3A mutation, 2 patients had IDH2 mutation, 1 patient had TET2 mutation, 3 patients had RUNX1 mutation and 1 patient had U2AF1 mutation.

Table 2. Clinical characteristics of patients 


\begin{tabular}{|c|c|c|c|c|c|c|c|c|c|c|c|}
\hline $\begin{array}{l}\text { Patient } \\
\text { no. }\end{array}$ & $\begin{array}{l}\text { Age } \\
\text { (years) }\end{array}$ & Gender & $\begin{array}{l}\text { WHO } \\
\text { diagnosis }\end{array}$ & $\begin{array}{l}\text { ECOG } \\
\text { PS } \\
\text { scores }\end{array}$ & $\begin{array}{l}\text { Blast } \\
\text { Percentage } \\
(\%)\end{array}$ & WBC $\left({ }^{*} 10^{9} / \mathrm{L}\right)$ & $\mathrm{HB}(\mathrm{g} / \mathrm{L})$ & $\operatorname{PLT}\left(* 10^{9} / \mathrm{L}\right)$ & $\begin{array}{l}\text { LDH } \\
\text { level } \\
\text { (IU/L) }\end{array}$ & $\begin{array}{l}\text { Mutated } \\
\text { gene }\end{array}$ & Cytogenetics \\
\hline 1 & 69 & $\mathrm{~F}$ & $\begin{array}{l}\text { AML-M5 } \\
\text { (second } \\
\text { relapse) }\end{array}$ & 2 & 14 & 0.9 & 80 & 65 & 144 & $\begin{array}{l}\text { CEBPA } \\
\text { NPM10 } \\
\text { TET2! } \\
\text { TP53! } \\
\text { DNMT3A }\end{array}$ & $46 \unrhd X X$ \\
\hline 2 & 71 & $\mathrm{~F}$ & $\begin{array}{l}\text { AML-M2 } \\
\text { (second } \\
\text { relapse) }\end{array}$ & 2 & 7.5 & 1.88 & 130 & 134 & 113 & IDH2 & $46, x X$ \\
\hline 3 & 74 & $M$ & AML-MRC & 3 & 20.5 & 3.45 & 51 & 263 & 317 & $\begin{array}{l}\text { ASXL1] } \\
\text { BCOR] } \\
\text { RUNX1] } \\
\text { IDH2 }\end{array}$ & $46, X Y$ \\
\hline 4 & 82 & $M$ & AML-MRC & 3 & 21 & 4.62 & 82 & 25 & 312 & $\begin{array}{l}\text { TP53[ } \\
\text { DNMT3A }\end{array}$ & $\begin{array}{l}\text { 46,XY, add(4)(p1। } \\
\text { del(5)(q21), del(7) } \\
\text { (q31), }+8, \text { del(16) } \\
\text { (q22) }\end{array}$ \\
\hline 5 & 71 & $M$ & AML-MRC & 3 & 20 & 1.83 & 72 & 2 & 129 & $\begin{array}{l}\text { RUNX } \\
\text { U2AF10 } \\
\text { FLT3-ITD }\end{array}$ & $46, X Y$ \\
\hline 6 & 65 & $\mathrm{~F}$ & AML-MRC & 3 & 37 & 1.3 & 65 & 298 & 473 & $\begin{array}{l}\text { IDH10 } \\
\text { NPM1 } \\
\text { CEBPA } \\
\text { DNMT3A }\end{array}$ & $46, x x$ \\
\hline 7 & 69 & $M$ & AML-MRC & 2 & 2.5 & 5.38 & 118 & 159 & 152 & TP53 & $\begin{array}{l}45, \mathrm{XY},-5, \operatorname{add}(11) \\
\text { (p15), add(13) } \\
\text { (p11),add(15) } \\
\text { (p11),-17,-18,-20, }\end{array}$ \\
\hline 8 & 68 & M & $\begin{array}{l}\text { AML- } \\
\text { MRC(relapse) }\end{array}$ & 2 & 18 & 2.04 & 124 & 81 & 240 & $\begin{array}{l}\text { RUNX1 } \\
\text { SRSF2 } \\
\text { IDH1 } \\
\text { BCOR } \\
\text { ASXL1 } \\
\text { SETBP1 } \\
\text { KMT2A } \\
\text { TET2 }\end{array}$ & $47 \rrbracket X Y \rrbracket+8$ \\
\hline 9 & 65 & $M$ & $\begin{array}{l}\text { AML- } \\
\text { MRC(relapse) }\end{array}$ & 2 & 13 & 5.48 & 157 & 83 & 182 & $\begin{array}{l}\text { ASXL1 } \\
\text { SRSF2 } \\
\text { GATA2 }\end{array}$ & $46, X Y$ \\
\hline
\end{tabular}

2. Adverse Events: Gastrointestinal reactions: 6/9 patients had mild nausea, no vomiting. Hematologic toxicity: Granulocytopenia occurred in $8 / 9$ patients during the first two cycles of treatment, of which 5 patients developed severe infections. One patient died of severe neutropenia and septic shock. Thrombcytopenia occurred in $5 / 9$ patients, of which 2 patients developed severe bleeding and died. There was no obvious tumor lysis syndrome in nine patients.(see table.3)

Table.3

3.Therapeutic effect: Except case 6 died in the early stage and the therapeutic effect could not be evaluated , 6 patients among the remaining 8 patients had efficacy with the response rate (CR/CRi) at 75\%, which occurred around the time when the regimen was given for 1.5 cycles(1-3cycles).

In case 1, the course of AML was 4 years. At the time of initial diagnosis, due to biallelic mutated CEBPA and mutated NPM1 without FLT3-ITD, the risk stratification was low-risk. At that time, standard induction therapy, medium and large dose of cytarabine consolidation, and intensive therapy were given, and the mutation gene was corrected after therapy. One year later, the patient relapsed for the first time, with mutations of TET2, TP53 (VAF=4.55\%) and DNMT3A genes. The patient was treated with low-dose chemotherapy containing another HMA, decitabine, and bone marrow obtained CR again. The second complete 


\begin{tabular}{|c|c|c|c|c|c|}
\hline \multirow[t]{2}{*}{$\begin{array}{l}\text { Adverse } \\
\text { Events }\end{array}$} & \multicolumn{2}{|l|}{ Hematologic toxicity } & \multicolumn{2}{|c|}{$\begin{array}{l}\text { Gastrointestinal } \\
\text { reactions }\end{array}$} & \multirow[t]{2}{*}{$\begin{array}{l}\text { Tumor lysis } \\
\text { syndrome }\end{array}$} \\
\hline & Granulocytopenia & Thrombcytopenia & Nausea & Vomiting & \\
\hline No.1 & yes & no & slight & no & no \\
\hline No.2 & yes & no & slight & no & no \\
\hline No.3 & $\begin{array}{l}\text { yes『skin and soft tissue } \\
\text { infectiongs,staphylococcemia }\end{array}$ & no & no & no & no \\
\hline No.4 & yes囚pneumonia & yes & slight & no & no \\
\hline No.5 & yes, pseudomonas aeruginosa & $\begin{array}{l}\text { Yes } ₫ \text { gastrointestinal } \\
\text { bleeding }\end{array}$ & slight & no & no \\
\hline No.6 & Yes,stenotrophomonas maltophilia pneumonia & Yes囚abdominal hemorrhage & slight & no & uncertain \\
\hline No.7 & no & yes & no & no & no \\
\hline No.8 & yes & no & slight & no & no \\
\hline No.9 & Yes,fungal pneumonia & yes & no & no & no \\
\hline
\end{tabular}

remission lasted for one year. Aside from the TET2 gene, TP53, DNMT3A, and NPM1 gene mutations occurred again in the second relapse, which was not relieved after 2 cycles of azacytidine treatment alone. The third CR (CR3) was achieved after 2 cycles of AZA+VEN treatment, and NPM1 mutation was corrected again.

Case 2 was initially diagnosed as a moderate risk patient. Standard induction chemotherapy and intensive treatment with high dose cytarabine was used in a consolidation period. After 6 years of long-term remission, azacytidine combined with low-dose cytarabine induction treatment was effective in obtaining CR2 after the first relapse occurred. During single drug azacytidine maintenance treatment, the patient relapsed again with $I D H 2$ gene mutation. Because an IDH2 inhibitor was not available, AZA+VEN treatment was administered, and CR3 was obtained after one cycle of treatment. The IDH2 gene mutation was corrected after two cycles.

Case 3 was diagnosed as AML-MRC with ASXL1, BCOR, RUNX1 and IDH2 gene mutations. According to the National Comprehensive Cancer Network (NCCN) guidelines, azacytidine with $75 \mathrm{mg} / \mathrm{m}^{2} / \mathrm{d}$ monotherapy was the first line choice. After four cycles of treatment, the proportion of bone marrow blast cells failed to decrease, and the patient had to continuously receive the transfusion of red blood cells. Therefore, the evaluation of curative effect was invalid and the paitne became a refractory case. After adjusting to AZA+VEN, CR1 was obtained after two cycles of treatment, and the mutation gene BCOR was resolved. These three patients received a combined VEN therapy after ineffective single drug azacytidine treatment, and achieved CR.

Case 4 was an elderly patient with complex karyotype with 7q- and TP53 gene mutation. Three factors including elder, complex karyotype, and TP53 mutation were independent adverse prognostic factors ${ }^{(13)}$. Therefore, AZA combined with VEN induction therapy was given. CRi was obtained after one course of treatment, and the VAF of TP53 decreased from $41.4 \%$ to $2.06 \%$.

Cases 5 and 6 were both initially diagnosed as MDS-EB2. After a course of single drug azacytidine treatment, the disease progressed to AML-MRC, and severe neutropenia occurred after the addition of venetoclax. The former case developed Pseudomonas aeruginosa, which improved after antibiotic treatment. But after another course of AZA+VEN treatment, there was no improvement and the patient died of gastrointestinal bleeding because of thrombocytopenia for long time

Case 6 developed neutropenia, Pseudomonas maltophilia infection and primary resistance to carbapenems. On the 14th day after treatment with AZA+VEN, the patient died of severe pulmonary infection.

Case 7 was diagnosed as AML-MRC with complex karyotype and TP53 mutation $\triangle \mathrm{VAF}$ is $58.1 \%$. Although CR1 was obtained by azacytidine combined with low dose cytarabine, the VAF value of TP53 gradually increased from $6.3 \%$ to $11.8 \%$ during consolidation treatment, suggesting that this case would be refractory to the therapies and the prognosis would be poor. After three cycles of combined therapy using AZA+VEN, the VAF value of TP53 increased from $11.8 \%$ to $28 \%$. Thrombocytopenia occurred more than 2 weeks after the fourth cycle treatment and died of abdominal hemorrhage.

Cases 8 and 9 were both diagnosed as AML-MRC and were treated with AZA and low-dose cytarabine when relapsed. After VEN be added for only one cycle, CR2 was obtained. In case 8, there were seven genes mutation when relapsed, including RUNX1, SRSF2, IDH1, ASXL1, SETBP1, KMT2A, TET2. When 2 cycles treatment finished, the mutation genes RUNX1, IDH1, ASXL1 and SETBP1 were resolved. There were three genes mutation including ASXL1, SRSF2 and GATA2 when relapsed in case 9 and only GATA2 mutation retained after two cycles treatment.

\section{Discussion}

DNA methylation and other epigenetic changes are involved in the pathogenesis of elderly AML. HMA has become the first choice for newly diagnosed elderly patients who are not suitable for high-intensity chemotherapy and hematopoietic stem cell transplantation and for R/R elderly AML patients. A retrospective study conducted in MD Anderson Cancer Center showed that the CR/ CRi rate was $28 \%$ in demethylation alone group and $56 \%$ in group using Bcl-2 inhibitor 
combined with demethylation after 1-3 courses of induction therapy ${ }^{(14)}$. These data demonstrate that Bcl-2 inhibitor combined with a demethylation drug is more effective than a demethylation drug alone.

All cases we studied were elderly frailty AML patients, including 5 R/R patients. Cases 1 and 2 were in their second relapse. They had used anthracyclines and cytarabine and other standard and high-dose chemotherapy regimens for many times in the past, but because of the organ function, they failed to continue the conventional chemotherapies. Most of the cases in this group had grade 3-4 myelosuppression after using VEN with 100-400 mg per day combined with azacytidine $75 \mathrm{mg} / \mathrm{m}^{2} / \mathrm{d}$. This dose is the same as that of adults.In the first course of treatment, neutropenia lasted for $6-15$ days. Six out of the 8 patients (75\%) showed significant therapeutic effect, CR/CRi, especially in all $5 \mathrm{R} / \mathrm{R}$ patients.

It has been previously reported that AZA+VEN shows better efficacy in low and medium-risk groups than in high-risk group according to genetic stratification ${ }^{(15)}$.In our study, 2/9 cases were in the medium and low risk groups at the time of initial diagnosis,whose PFS are 16 and 13 months respectively up to date. Studies on gene mutations have shown that AML patients with RUNX1 and IDH2 mutations had relatively better response to

AZA+VEN combinations ${ }^{(16,17)}$. Three cases tested positive for IDH2 or RUNX1 mutations, which is consistent with the findings in the aforementioned study ${ }^{(16)}$. A retrospective clinical study showed that patients with FLT3-ITD, TP53, and N/KRAS gene mutations were more likely to have no response or relapse, while $21 \%$ of patients who received venetoclax combined with HAM rescue treatment had response, and the median OS was longer than those who did not receive rescue treatment (2.9 months vs 1.3 months) ${ }^{(18)}$. In our study, TP53 mutation was found in cases 1, 4 and 7 before treatment. Up to the followup period, case 1 had achieved PFS of 16 months. The overall survival (OS) of case 4 was 11 months, which was significantly longer than the reported median OS of elderly AML ${ }^{(3)}$, but PFS was only 2 months. Case 7 died recently owing to abdominal hemorrhage, OS is up to 7 months. The VAF value of TP53 mutation of case 7 did not decrease after the addition of VEN. It seems that compared with AZA monotherapy, the addition of VEN does not improve the prognosis of patients. Because the patients with TP53 mutation had poor response to conventional chemotherapy, and the median survival time was $5-9$ months ${ }^{(19)}$. The fact that case 1 is in continuous CR for 16 months and the latter two cases have the short remission time may be related to complex karyotype, and high VAF value ${ }^{(20)}$.Case4 and case 7 both have complex karyotype including $-5 / 5 q$ - and/or $-7 / 7 q$ - chromosomal deletions and TP53 mutation with VAF $>20 \%$.. More cases are needed for further study for VAF of TP53 and prognosis.

There was no tumor lysis in any of the 8 patients, which was related to the low proportion of primordial cells in patients before starting the treatment. However, early death occurred in one case (case 6), related to the duration of neutropenia longer than 2 weeks, infection, and potential tumor lysis. In the treatment of AML with VEN, higher peripheral blood leukocyte counts, blood lactate dehydrogenase (LDH) levels, and precursor cell counts in bone marrow or peripheral blood were associated with higher mortality rates, ${ }^{(21)}$.

Our study has several limitations. First, the cohort of patients analyzed is relatively small. It is worth reporting because few studies about AZA+VEN in the treatment of elderly R/R AML in Asian population has been reported. In our study, 8/9(89\%) patients relapsed or progressed in the stage of AZA monothrapy, The therapeutic effect of AZA+VEN was significantly improved compared with azacytidine alone, and the overall safety was consistent with previous studies ${ }^{(10)}$. However, it needs to be cautioned that serious myelosuppression could happen. It is noted in our study that the lack of granulocyte is the major adverse effect for the combination therapy, which may lead to early death of the patients. The initial result from this small cohort study is encouraging. With venetoclax entering the Chinese market, further extended studies are needed to optimize the therapeutic regimen, as well as to characterize the mechanism of action of this combination therapy for the elderly R/R AML patients.

\section{Conclusion}

Venetoclax combined with azacytidine regimen in elderly R/R AML patients is an effective and well tolerated rescue scheme . 75\% patients got response. Especially, all the 5 relapsed patients got CR/CRi. The patients with TP53 mutation with lower VAF may be benifit from VEN and AZA.

\section{Abbreviations}

AML: acute myeloid leukemia; R/R: relapsed and refractory; MRC: myelodysplasia-related changes ; CR: complete response; CRi:complete response with incomplete hematologic recovery; PFS: progression free remission; VAF: variant allele fraction value; DOR: duration of remission; PS: performance status; OS: overall survival; HMA: hypomethylation agents; VEN: venetoclax; Bcl-2: B cell leukemia/lymphoma-2; AZA :azacytidine; ELN: European LeukemiaNet; ORR: overall response rate; EDTA囚Ethylene Diamine Tetraacetic Acid; PCR: polymerase chain reaction; CYP: cytochrome P450; NCCN: national comprehensive cancer network; MDS: myelodysplastic syndrome; LDH: lactate dehydrogenase;

\section{Declarations}

\section{Ethics approval and consent to participate}

This research was supported by Beijing Natural Science Foundation. (Z200022). The retrospective study was a part of this research,and ethics has been approved. Informed consent was signed by all participants. All procedures performed in studies involving human participants were in accordance with the ethical standards of the institutional and/or national research committee and with the 1964 Helsinki declaration and its later amendments or comparable ethical standards.

\section{Consent for publication}


All authors agreed to publish.

\section{Availability of data and material}

All data are available. Identifying images or other personal or clinical details can be obtained from all of the participants..

\section{Competing interests}

The authors have no conflict of interest to declare.

\section{Funding}

This work was supported by Beijing Natural Science Foundation. (Z200022\ The funder,WLS, who was also the corresponding author of the article, conceived and designed the research;

\section{Authors' contributions}

LS and WLS conceived and designed the research. RHH wrote the main manuscript text. All cases were provided by XXL, XLC, WHH, HZ, YXG, GXW. WLS reviewed the manuscript.All authors have read and approved the manuscript.

\section{Acknowledgements}

The authors thank QM,CYL and JJH for participating in the lab exercises

\section{References}

1. Howlader N, Noone AM, Krapcho M, Miller D, Brest A, Yu M,. SEER Cancer statistics review, 1975-2016. Bethesda, MD: National Cancer Institute; 2019https:// seer.cancer.gov/csr/1975_2016/ [based on November 2018 SEER data submission, posted to the SEER web site, April 2019. Accessed 4/18/2019].

2. Appelbaum FR, Gundacker H, Head DR, Slovak ML, Willman CL, Godwin JE, Anderson JE, Petersdorf SH. Age and acute myeloid leukemia. Blood. 2006 May 1;107(9):3481-5

3. Shallis RM, Wang R, Davidoff A, Ma X, Zeidan AM. Epidemiology of acute myeloid leukemia: Recent progress and enduring challenges. Blood Rev. 2019 Jul;36:70-87

4. Kantarjian HM, Thomas XG, Dmoszynska A, Wierzbowska A, Mazur G, Mayer J, Gau JP, Chou WC, Buckstein R, Cermak J, Kuo CY, Oriol A, Ravandi F, Faderl S, Delaunay J, Lysák D, Minden M, Arthur C. Multicenter, randomized, open-label, phase III trial of decitabine versus patient choice, with physician advice, of either supportive care or low-dose cytarabine for the treatment of older patients with newly diagnosed acute myeloid leukemia. J Clin Oncol. 2012 Jul 20;30(21):2670-7.

5. Gardin C, Dombret H. Hypomethylating Agents as a Therapy for AML. Curr Hematol Malig Rep. 2017 Feb;12(1):1-10.

6. Deng J, Isik E, Fernandes SM, Brown JR, Letai A, Davids MS. Bruton's tyrosine kinase inhibition increases BCL-2 dependence and enhances sensitivity to venetoclax in chronic lymphocytic leukemia. Leukemia. 2017 Oct;31(10):2075-2084.

7. Bose P, Gandhi V, Konopleva M. Pathways and mechanisms of venetoclax resistance. Leuk Lymphoma. 2017 Sep;58(9):1-17.

8. .Konopleva M, Pollyea DA, Potluri J, Chyla B, Hogdal L, Busman T, McKeegan E, Salem AH, Zhu M, Ricker JL, Blum W, DiNardo CD, Kadia T, Dunbar M, Kirby R, Falotico N, Leverson J, Humerickhouse R, Mabry M, Stone R, Kantarjian H, Letai A. Efficacy and Biological Correlates of Response in a Phase II Study of Venetoclax Monotherapy in Patients with Acute Myelogenous Leukemia. Cancer Discov. 2016 Oct;6(10):1106-1117.

9. Bogenberger JM, Delman D, Hansen N, Valdez R, Fauble V, Mesa RA, Tibes R. Ex vivo activity of BCL-2 family inhibitors ABT-199 and ABT-737 combined with 5-azacytidine in myeloid malignancies. Leuk Lymphoma. 2015 Jan;56(1):226-9.

10. DiNardo CD, Maiti A, Rausch CR, Pemmaraju N, Naqvi K, Daver NG, Kadia TM, Borthakur G, Ohanian M, Alvarado Y, Issa GC, Montalban-Bravo G, Short NJ, Yilmaz M, Bose P, Jabbour EJ, Takahashi K, Burger JA, Garcia-Manero G, Jain N, Kornblau SM, Thompson PA, Estrov Z, Masarova L, Sasaki K, Verstovsek S, Ferrajoli A, Weirda WG, Wang SA, Konoplev S, Chen Z, Pierce SA, Ning J, Qiao W, Ravandi F, Andreeff M, Welch JS, Kantarjian HM, Konopleva MY. 10-day decitabine with venetoclax for newly diagnosed intensive chemotherapy ineligible, and relapsed or refractory acute myeloid leukaemia: a single-centre, phase 2 trial. Lancet Haematol. 2020 Oct;7(10):e724-e736.

11. Döhner H, Estey E, Grimwade D, Amadori S, Appelbaum FR, Büchner T, Dombret H, Ebert BL, Fenaux P, Larson RA, Levine RL, Lo-Coco F, Naoe T, Niederwieser D, Ossenkoppele GJ, Sanz M, Sierra J, Tallman MS, Tien HF, Wei AH, Löwenberg B, Bloomfield CD. Diagnosis and management of AML in adults: 2017 ELN recommendations from an international expert panel. Blood. 2017 Jan 26;129(4):424-447.

12. Jonas BA, Pollyea DA. How we use venetoclax with hypomethylating agents for the treatment of newly diagnosed patients with acute myeloid leukemia. Leukemia. 2019;33(12):2795-2804.

13. Papaemmanuil E, Gerstung M, Bullinger L, Gaidzik VI, Paschka P, Roberts ND, Potter NE, Heuser M, Thol F, Bolli N, Gundem G, Van Loo P, Martincorena I, Ganly P, Mudie L, McLaren S, O'Meara S, Raine K, Jones DR, Teague JW, Butler AP, Greaves MF, Ganser A, Döhner K, Schlenk RF, Döhner H, Campbell PJ. Genomic Classification and Prognosis in Acute Myeloid Leukemia. N Engl J Med. 2016 Jun 9;374(23):2209-2221.

14. Lachowiez CA, Loghavi S, Kadia TM, Daver N, Borthakur G, Pemmaraju N, Naqvi K, Alvarado Y, Yilmaz M, Short N, Ohanian M, Pierce SR, Patel KP, Qiao W, Ning J, Sasaki K, Takahashi K, Jabbour E, Andreeff M, Ravandi F, Kantarjian HM, Konopleva M, DiNardo CD. Outcomes of older patients with NPM1- 
mutated AML: current treatments and the promise of venetoclax-based regimens. Blood Adv. 2020 Apr 14;4(7):1311-1320.

15. Aldoss I, Yang D, Pillai R, Sanchez JF, Mei M, Aribi A, Ali H, Sandhu K, Al Malki MM, Salhotra A, Khaled S, Sun W, O'Donnell M, Snyder D, Nakamura R, Stein AS, Forman SJ, Marcucci G, Pullarkat V. Association of leukemia genetics with response to venetoclax and hypomethylating agents in relapsed/refractory acute myeloid leukemia. Am J Hematol. 2019 Oct;94(10):E253-E255.

16. DiNardo CD, Rausch CR, Benton C, Kadia T, Jain N, Pemmaraju N, Daver N, Covert W, Marx KR, Mace M, Jabbour E, Cortes J, Garcia-Manero G, Ravandi F, Bhalla KN, Kantarjian H, Konopleva M. Clinical experience with the BCL2-inhibitor venetoclax in combination therapy for relapsed and refractory acute myeloid leukemia and related myeloid malignancies. Am J Hematol. 2018 Mar;93(3):401-407.

17. Chan SM, Thomas D, Corces-Zimmerman MR, Xavy S, Rastogi S, Hong WJ, Zhao F, Medeiros BC, Tyvoll DA, Majeti R. Isocitrate dehydrogenase 1 and 2 mutations induce BCL-2 dependence in acute myeloid leukemia. Nat Med. 2015 Feb;21(2):178 - 84.

18. Maiti A, Rausch CR, Cortes JE, Pemmaraju N, Daver NG, Ravandi F, Garcia-Manero G, Borthakur G, Naqvi K, Ohanian M, Short NJ, Alvarado Y, Kadia TM, Takahashi K, Yilmaz M, Jain N, Kornblau S, Montalban Bravo G, Sasaki K, Andreeff M, Bose P, Ferrajoli A, Issa GC, Jabbour EJ, Masarova L, Thompson PA, Wang S, Konoplev S, Pierce SA, Ning J, Qiao W, Welch JS, Kantarjian HM, DiNardo CD, Konopleva MY. Outcomes of relapsed or refractory acute myeloid leukemia after frontline hypomethylating agent and venetoclax regimens. Haematologica. 2021 Mar 1;106(3):894-898. 19.Welch JS. Patterns of mutations in TP53 mutated AML. Best Pract Res Clin Haematol. 2018 Dec;31(4):379-383.

19. Hong M, Zhu H, Sun Q, Zhu Y, Miao Y, Yang H, Qiu HR, Li JY, Qian SX. Decitabine in combination with low-dose cytarabine, aclarubicin and G-CSF tends to improve prognosis in elderly patients with high-risk AML. Aging (Albany NY). 2020 Apr 1;12(7):5792-5811.

20. Ram R, Amit O, Zuckerman T, Gurion R, Raanani P, Bar-On Y, Avivi I, Wolach O. Venetoclax in patients with acute myeloid leukemia refractory to hypomethylating agents-a multicenter historical prospective study. Ann Hematol. 2019 Aug;98(8):1927-1932. 